\title{
ON THE SEASONAL OCCURRENCE OF APPENDICULARIANS IN WATERS OFF THE COAST OF SÃO PAULO STATE
}

(Received in 10/8/1970)

\author{
T. M. Tundisi
}

Instituto Oceanográfico da Universidade de São Paulo

\section{SYNOPSIS}

Studies were made on the seasonal variation and qualitative composition of the Appendicularia population from a limited area of the South Atlantic $25^{\circ} 09.5^{\prime} \mathrm{S}$ to $24^{\circ} 04.0^{\prime} \mathrm{S}$ latitude and $47^{\circ} 48.4^{\prime} \mathrm{W}$ to $46^{\circ} 00.4^{\prime} \mathrm{W}$ longitude at the São Paulo State coast off Cananéia and off Santos.

The pattern of the seasonal variation was irregular for the most important species $(O$. longicauda). Some qualitative differences regarding the species composition are discussed.

\section{INTRODUCTION}

Studies on distribution, specific composition, and abundance of Appendicularia from the South Atlantic have been made by several authors, namely LOHMANN (1896, 1931), Lohmann \& Buckmann (1926), Lohmann \& Hentschel (1939), Garstang \& Georgeson (1935), Udvardy (1958) and Fenaux (1967).

Papers by BJörNBERG \& ForNERIS (1956a, 1956b, 1958) and ForNERIS (1965) give some indication of the association of certain species to some water-masses.

In the present note, an attempt has been made to indicate the seasonal variations of the Appendicularia population, from a limited region of the South Atlantic: $25^{\circ} 09.5 \mathrm{~S}$ to $24^{\circ} 04.0^{\prime} \mathrm{S}$ latitude and $47^{\circ} 48.4^{\prime} \mathrm{W}$ to $46^{\circ} 00.4$ 'W longitude. Also the relative abundance of the different species was studied.

\section{MATERIAL AND METHODS}

Three oceanographic stations were established in two regions off Cananéia and off Santos. Sampling was made fortnighthly during 1958, 1959 and 1960 off Cananéia, and during 1960 at the same time intervals off Santos.

Station position, local depth, and sampling depths are given in Table I.

Plankton samples were collected vertically with a Hensen net, with a mesh size aperture of $295 \mu$.

Publ. n.० 300 do Inst. Ocean. da USP.
They were fixed with formalin, $4 \%$ and examined with a binocular with magnification of 96x. All the results were calculated as number of individuals $/ \mathrm{m}^{3}$.

Water temperature was measured with reversing thermometers and salinity was determined with the Knudsen method. These two parameters were measured at surface and at the sampling depths given in Table I.

\section{NOTES ON ENVIRONMENTAL DATA}

The coast of the State of São Paulo was under the influence of three types of water masses. These waters were characterized by the following situations regarding temperature and salinity data (EMILson $\cdot 1959,1961)$.
1) "Coastal waters"
Salinity
Temperature
2) "Shelf waters"

$$
<35 \%
$$$$
>19^{\circ} \mathrm{C}
$$
3) "Tropical waters"

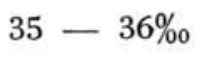$$
<22^{\circ} \mathrm{C}
$$

"Coastal water" was present at the three stations off Cananéia. Occasionally "shelf water" was found at station III. This water type was quite constant off Santos but occasionally "tropical water" occurred (LeINebö, 1969).

Temperature and salinity average monthly values for each station are given on Tables II and III. 
TABLE $\overline{\mathrm{I}}$ - Station position and sampling depths

\begin{tabular}{|c|c|c|c|c|c|}
\hline Region & Station & Position & Distance from the coast (n.m.) & $\begin{array}{l}\text { Sampling depth } \\
(\mathrm{m})\end{array}$ & $\begin{array}{l}\text { Local depth } \\
\text { (m) }\end{array}$ \\
\hline \multirow{3}{*}{$\begin{array}{l}\text { Off } \\
\text { Cananéia }\end{array}$} & I & $\begin{array}{l}25^{\circ} 07.9^{\prime} \mathrm{S} \\
47^{\circ} 48.4^{\prime} \mathrm{W}\end{array}$ & 5.6 from Bom Abrigo & 15 & 19 \\
\hline & II & $\begin{array}{l}25^{\circ} 08.4^{\prime} \mathrm{S} \\
47^{\circ} 44.2^{\prime} \mathrm{W}\end{array}$ & 9.5 from Bom Abrigo & 15 & 20 \\
\hline & III & $\begin{array}{l}25^{\circ} 09.5^{\prime} \mathrm{S} \\
47^{\circ} 35.7^{\prime} \mathrm{W}\end{array}$ & 17.2 from Bom Abrigo & 25 & 30 \\
\hline \multirow{3}{*}{$\begin{array}{l}\text { Off } \\
\text { Santos }\end{array}$} & I & $\begin{array}{l}24^{\circ} 04.8^{\prime} \mathrm{S} \\
26^{\circ} 13.7^{\prime} \mathrm{W}\end{array}$ & 5.0 from Ilha da Moela & 25 & 28 \\
\hline & II & $\begin{array}{l}24^{\circ} 10.0^{\prime} \mathrm{S} \\
46^{\circ} 08.0^{\prime} \mathrm{W}\end{array}$ & 10.0 from Ilha da Moela & 35 & 37 \\
\hline & III & $\begin{array}{l}24^{\circ} 16.8^{\prime} \mathrm{S} \\
46^{\circ} 00.4^{\prime} \mathrm{W}\end{array}$ & 20.0 from Ilha da Moela & 45 & 49 \\
\hline
\end{tabular}

\section{RESULTS AND DISCUSSION}

\section{a) Considerations on Appendicularia families}

In the area under investigation three families of Appendicularia with following genus and species were found (FEnaux, 1963):

Family Oikopleuridae

Genus Oikopleura Mertens

O. longicauda Vogt

$O$. fusiformis f. typica Fol

$O$. fusiformis f. cornutogastra Aida

O. dioica $\mathrm{Fol}$

O. rufescens $\mathrm{Fol}$

Genus Stegosoma Chun

Stegosoma magnun Langerhans

Family Fritillariidae

Genus Fritillaria Quoy \& Gaimard

F. haplostoma Fol

$F$. pellucida Busch

F. formica f. digitata Lohmann \&

Buckmann

F. formica f. tuberculata Lohmann \&

$F$. borealis f. intermedia Lohmann

$F$. borealis $\mathrm{f}$. sargassi Lohmann

Genus Appendicularia Fol

Appendicularia sicula Fol

Family Kowalevskiidae

Genus Kowalevskia Fol

Kowalevskia tenuis Fol
Studies on appendicularians from other regions have shown that the family Oikopleuridae constitutes the bulk of its population. The material studied showed that the family Oikopleuridae dominates the samples with a percentage of $85-99 \%$ of the total of Appendicularia. The other two families Fritillariidae and Kowalevskiidae were relatively poorly represented. This result seems to be a characteristic of tropical waters (Fenaux, 1969). However, at regions with a distinct seasonal cycle a succession in the relative abundance of the three families can be found (Fenaux 1959, 1963).

\section{b) Relative abundance of the species}

Qualitative and quantitative differences in species composition were found at the three stations studied off Cananéia and off Santos (Table IV).

Station I off Cananéia nearest to the coast showed a slight reduction in number of species and the occurrence of species from the genus Fritillaria was sporadic. Certain influences of the waters of the lagunar region of Cananéia may probably occur at this station (Tavares, 1967). The three stations situated off Santos, showed no difference in specific composition of its Appendicularia fauna.

A small variation on the total density of Appendicularia at the three stations off Cananéia was found, except for station I (1958). Off Santos stations I and II showed a density relatively larger than the stations off Cananéia.

From the eleven species present, $O$. longicauda was the commonest, with a percentage of $71.2 \%$ of 


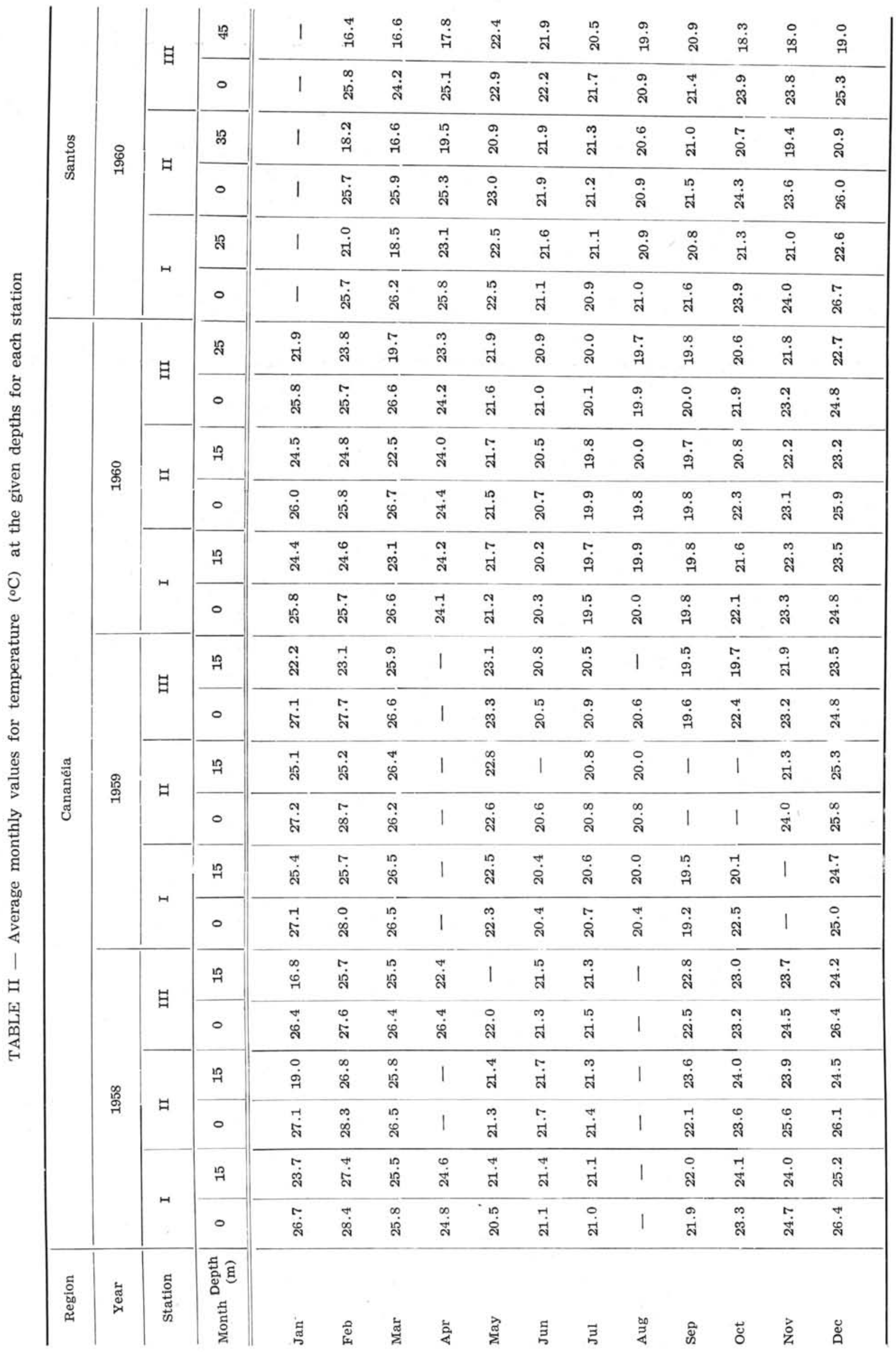




\begin{tabular}{|c|c|c|c|c|c|c|c|c|c|c|c|c|c|c|c|}
\hline \multirow{6}{*}{ 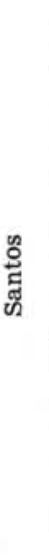 } & \multirow{6}{*}{ ‡్ } & \multirow{2}{*}{ 当 } & \& & 1 & $\begin{array}{l}\text { \% } \\
\text { : }\end{array}$ & 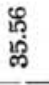 & 范 & 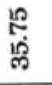 & 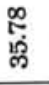 & 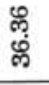 & 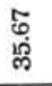 & 哭 & 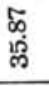 & 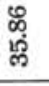 & $\begin{array}{l}\text { 量 } \\
\text { 㔽 }\end{array}$ \\
\hline & & & 0 & 1 & 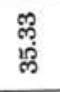 & 落 & 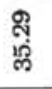 & 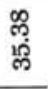 & 霑 & đ్ & 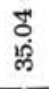 & $\begin{array}{l}\vec{p} \\
\text { p. } \\
\text { p. }\end{array}$ & 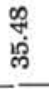 & 点 & 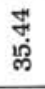 \\
\hline & & \multirow{2}{*}{$\exists$} & ळू & 1 & $\begin{array}{l}\text { 岕 } \\
\dot{\phi}\end{array}$ & 槅 & 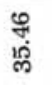 & $\begin{array}{l}\text { 苛 } \\
\text { 点 }\end{array}$ & స్్ㅐ & $\begin{array}{l}\text { 营 } \\
\text { 号 }\end{array}$ & 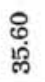 & 疍 & 总 & 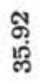 & $\begin{array}{l}\text { 㔛 } \\
\text { }\end{array}$ \\
\hline & & & 0 & 1 & 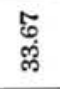 & 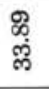 & $\underset{\vec{n}}{\infty}$ & 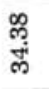 & 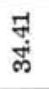 & శ్లే & 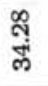 & స్ & $\begin{array}{l}\text { हु } \\
\text { हैं }\end{array}$ & స్ & 帒 \\
\hline & & \multirow{2}{*}{$H$} & In & 1 & $\begin{array}{l}\vec{m} \\
\text { }\end{array}$ & 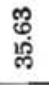 & 蔍 & స్ & 总 & 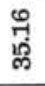 & 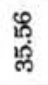 & 占 & $\begin{array}{l}\text { 㤩 } \\
\text { 峞 }\end{array}$ & 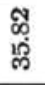 & 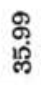 \\
\hline & & & 0 & 1 & స్ & $\begin{array}{l}\text { ज్ } \\
\text { f్ }\end{array}$ & ণ্ণ & 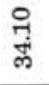 & 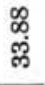 & 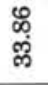 & 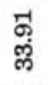 & 疍 & 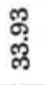 & 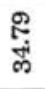 & స్ \\
\hline \multirow{18}{*}{ 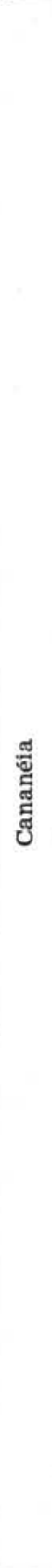 } & \multirow{6}{*}{ @ } & \multirow{2}{*}{ 百 } & $\mathscr{B}$ & 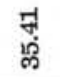 & 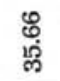 & 点 & ఝ్ & 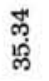 & $\begin{array}{c}\text { స్ } \\
\text { ले }\end{array}$ & 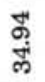 & 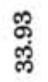 & †े & 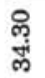 & 蛅 & 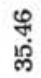 \\
\hline & & & 0 & $\begin{array}{l}\text { लू } \\
\text { लें }\end{array}$ & $\begin{array}{l}\text { ڤ̊. } \\
\text { 岗 }\end{array}$ & $\begin{array}{l}\text { 㳴 } \\
\text { कें }\end{array}$ & 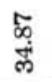 & $\begin{array}{l}\text { गे } \\
\text { लें }\end{array}$ & 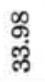 & $\begin{array}{l}\text { Iे } \\
\text { ले }\end{array}$ & $\begin{array}{l}\mathscr{d} \\
\stackrel{\infty}{0}\end{array}$ & न्ले & 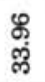 & I़े & 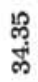 \\
\hline & & \multirow{2}{*}{$\exists$} & $\stackrel{10}{1}$ & సָำ & స్ં & $\begin{array}{l}\vec{p} \\
\text { p. }\end{array}$ & $\begin{array}{l}\overrightarrow{0} \\
\text { 苟 }\end{array}$ & 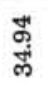 & $\begin{array}{l}\text { జ్ } \\
\text { लें }\end{array}$ & $\begin{array}{l}\vec{m} \\
\text { ले }\end{array}$ & $\begin{array}{l}8 \\
\text { में }\end{array}$ & హ్ల్ల & శ్ ఫే & 䨔 & $\begin{array}{l}\text { मृ } \\
\text { लें }\end{array}$ \\
\hline & & & 0 & స్ల్ల & 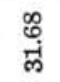 & శ్ర్లో & 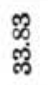 & $\begin{array}{l}\vec{b} \\
\text { लें }\end{array}$ & $\frac{\text { I্ }}{\text { Hें }}$ & 总 & $\begin{array}{l}\stackrel{J}{*} \\
\text { में }\end{array}$ & 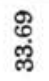 & $\begin{array}{l}\text { 点 } \\
\text { 岗 }\end{array}$ & స్ & ำ \\
\hline & & \multirow{2}{*}{$H$} & 足 & 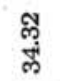 & लू & 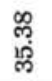 & নี & $\begin{array}{l}\vec{\infty} \\
\text { लें }\end{array}$ & 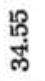 & $\begin{array}{l}\text { న్ర } \\
\text { गें }\end{array}$ & 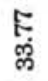 & 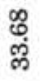 & 品 & ఫ్ల & 离 \\
\hline & & & 0 & 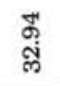 & 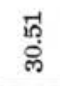 & $\begin{array}{l}\text { ్. } \\
\text { ه్ల }\end{array}$ & $\begin{array}{l}\text { క్ } \\
\text { लें }\end{array}$ & స్ & 怘 & 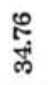 & 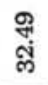 & 点 & 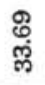 & 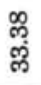 & $\begin{array}{l}\text { h. } \\
\text { d. }\end{array}$ \\
\hline & \multirow{6}{*}{ 兽 } & \multirow{2}{*}{ 目 } & 吾 & $\begin{array}{l}\tilde{h} \\
\text { 岗 }\end{array}$ & $\begin{array}{l}\text { జ్ } \\
\text { ஸీ }\end{array}$ & $\begin{array}{l}\text { స్ } \\
\text { ஸ్ }\end{array}$ & 1 & 营 & 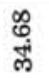 & 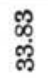 & 胥 & 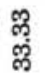 & $\begin{array}{l}\vec{m} \\
\text { ले }\end{array}$ & $\begin{array}{l}\text { मृ } \\
\text { लें }\end{array}$ & $\begin{array}{l}\text { t. } \\
\text { में }\end{array}$ \\
\hline & & & 0 & 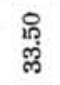 & జ్ల్ల & 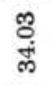 & 1 & 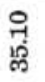 & 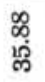 & $\underset{\varpi ్}{\mathscr{\varpi}}$ & న్ల్ & 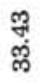 & జ్లి & 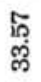 & 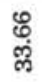 \\
\hline & & \multirow{2}{*}{$\exists$} & $\stackrel{20}{\pi}$ & 趈 & శ్రి & 点 & 1 & 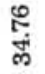 & 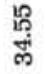 & $\begin{array}{l}\text { 吊 } \\
\text { ్ֻ }\end{array}$ & $\begin{array}{l}\text { శ్ } \\
\text { ల్ }\end{array}$ & 1 & 1 & $\underset{\infty}{~}$ & 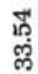 \\
\hline & & & 0 & $\begin{array}{l}\vec{m} \\
\text { జ్ }\end{array}$ & $\begin{array}{l}\text { 吊 } \\
\text { 亗 }\end{array}$ & $\begin{array}{l}\text { స్ట్ర } \\
\text { }\end{array}$ & 1 & 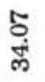 & $\begin{array}{l}\text { शे } \\
\text { ले }\end{array}$ & 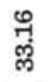 & $\begin{array}{l}\infty \\
\stackrel{\varpi}{\circ}\end{array}$ & 1 & 1 & 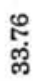 & $\begin{array}{l}\text { h్ } \\
\text { p. }\end{array}$ \\
\hline & & \multirow{2}{*}{$H$} & in & 年 & $\begin{array}{l}\text { 䓟 } \\
\text { लें }\end{array}$ & 哭 & 1 & 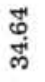 & $\begin{array}{l}\text { I্ } \\
\text { लें }\end{array}$ & 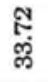 & $\underset{\infty}{\mathbb{~}}$ & 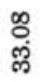 & $\begin{array}{l}\mathscr{m} \\
\dddot{m}\end{array}$ & 1 & $\begin{array}{l}\text { : } \\
\text { 吕 }\end{array}$ \\
\hline & & & 0 & 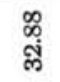 & 疍 & 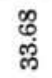 & 1 & 营 & 承 & స్లో & ণ్ & $\begin{array}{l}\text { స్ } \\
\text { స్ }\end{array}$ & జ్ల్ల & 1 & $\begin{array}{l}7 \\
\text { d }\end{array}$ \\
\hline & \multirow{6}{*}{ 吕 } & \multirow{2}{*}{ 白 } & $\stackrel{n}{n}$ & $\begin{array}{l}\text { मी } \\
\text { 苞 }\end{array}$ & $\begin{array}{l}\text { গู } \\
\text { 岗 }\end{array}$ & $\begin{array}{l}\mathscr{\$} \\
\text { ले }\end{array}$ & 哭 & 褐 & 옹 & $\begin{array}{l}\text {. } \\
\text { di }\end{array}$ & 1 & 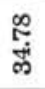 & 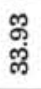 & $\begin{array}{l}\text {.0 } \\
\text { लें }\end{array}$ & 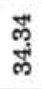 \\
\hline & & & 0 & 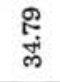 & 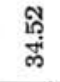 & 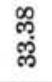 & స్ట్ర & 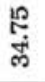 & 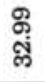 & 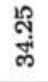 & 1 & $\begin{array}{l}8 \\
\text { गें }\end{array}$ & 范 & $\begin{array}{l}\mathscr{\infty} \\
\stackrel{\infty}{\infty}\end{array}$ & $\begin{array}{l}\text { t] } \\
\text { गे }\end{array}$ \\
\hline & & \multirow{2}{*}{$\exists$} & $\stackrel{12}{7}$ & $\begin{array}{l}\text { \&̊ } \\
\text { هึ }\end{array}$ & $\begin{array}{l}\text { शे } \\
\text { ले }\end{array}$ & $\begin{array}{l}\text { ஜ } \\
\text { लें }\end{array}$ & 1 & సึ & $\begin{array}{l}\overrightarrow{0} \\
\text { di } \\
\text { di }\end{array}$ & $\begin{array}{l}\text { पें } \\
\text { में }\end{array}$ & 1 & 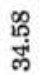 & జ్ఞ & 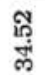 & సे \\
\hline & & & 0 & न्ల & 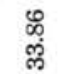 & $\begin{array}{l}\text { ఫ్ల } \\
\text { స్ల }\end{array}$ & 1 & 趈 & స్లు & 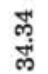 & 1 & స్లి & $\begin{array}{l}\text { ఝ్ల } \\
\text { స్ల }\end{array}$ & $\begin{array}{l}\text { జ్ } \\
\text { జ్ల }\end{array}$ & 륧 \\
\hline & & & ID & $\begin{array}{l}8 \\
\text { id }\end{array}$ & $\begin{array}{l}0 \\
\text { 10 }\end{array}$ & స్ & ஜ. & 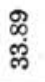 & 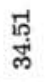 & 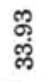 & 1 & శ్ & 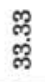 & శ్ ఫే & స్ల్ \\
\hline & & & 0 & 苚 & $\begin{array}{l}\stackrel{\infty}{\infty} \\
\stackrel{\infty}{\infty}\end{array}$ & శ్లి & 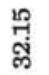 & ఊ్ర & స్ట్ & $\begin{array}{l}\text { ద్ } \\
\underset{\varpi}{0}\end{array}$ & 1 & ले & 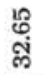 & జ్లై & స్ల్లో \\
\hline 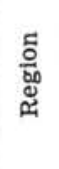 & ฮ્ભ & 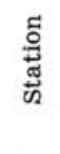 & 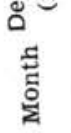 & 品 & 民ి & 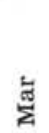 & $\frac{a}{4}$ & $\frac{\pi}{z}$ & 吉 & 寻 & $\frac{00}{4}$ & ๗ั. & $\overrightarrow{\mathrm{o}}$ & 总 & Дั \\
\hline
\end{tabular}


TABLE IV - The density of species expressed as number of organisms per cubic meter off Cananéia and off Santos

\begin{tabular}{|c|c|c|c|c|c|c|c|c|c|c|c|c|}
\hline \multirow{3}{*}{$\begin{array}{c}\text { Region } \\
\text { Year } \\
\text { Species }\end{array}$} & \multicolumn{9}{|c|}{ Cananéía } & \multirow{2}{*}{\multicolumn{3}{|c|}{$\begin{array}{c}\text { Santos } \\
1960\end{array}$}} \\
\hline & \multicolumn{3}{|c|}{1958} & \multicolumn{3}{|c|}{1959} & \multicolumn{3}{|c|}{1960} & & & \\
\hline & I & II & III & I & II & $\mathrm{III}$ & I & II & III & I & II & III \\
\hline o. longicauda & 61.7 & 80.4 & 104.7 & 128.1 & 116.1 & 81.3 & 92.8 & 92.2 & 83.2 & 143.1 & 149.3 & 82.2 \\
\hline o. fusiformis & 0.8 & 8.3 & 23.7 & 14.7 & 39.4 & 37.8 & 9.2 & 37.1 & 25.8 & 38.0 & 54.2 & 21.2 \\
\hline o. dioica & 11.6 & 7.8 & 0.1 & 11.0 & 10.5 & 0.9 & 15.3 & 20.9 & 4.2 & 32.9 & 1.4 & 1.2 \\
\hline o. rufescens & - & - & - & - & - & 0.2 & 0.4 & 2.2 & 2.9 & 0.6 & 0.8 & 0.4 \\
\hline F. haplostoma & 0.7 & 1.3 & 0.1 & 0.2 & 1.2 & 1.6 & 2.3 & 1.5 & 2.4 & 6.7 & 8.4 & 8.4 \\
\hline F. borealis & - & - & + & - & + & 0.7 & + & + & 1.4 & 1.4 & 3.3 & 8.2 \\
\hline F. pellucida & + & + & 0.2 & 0.1 & + & 2.3 & - & + & 0.4 & 0.4 & 1.7 & 1.2 \\
\hline F. formica & - & - & 0.1 & - & 0.1 & 0.8 & 0.5 & 1.0 & 5.4 & 0.4 & 1.0 & 0.4 \\
\hline K. tenuis & - & - & - & - & - & - & - & 0.3 & 0.4 & 0.4 & 0.8 & 0.3 \\
\hline A. sicula & + & + & - & - & - & - & - & - & + & - & + & + \\
\hline s. magnum & - & - & - & - & - & - & - & - & - & - & + & - \\
\hline Total & 74.8 & 97.8 & 128.9 & 154.1 & 167.3 & 125.6 & 120.5 & 155.2 & 126.1 & 223.9 & 220.9 & 123.5 \\
\hline
\end{tabular}

TABLE V - Percentage of the two forms of $O$. fusiformis (f. typica and f. cornutogastra) at the three stations off Cananéia and off Santos

\begin{tabular}{|c|c|c|c|c|}
\hline Cananéia & Species & Station I & Station II & Station III \\
\hline 1958 & $\begin{array}{l}\text { o. fusiformis f. typica } \\
\text { o. fusiformis f. cornutogastra }\end{array}$ & $\begin{array}{r}98 \% \\
2 \%\end{array}$ & $\begin{array}{r}96 \% \\
4 \%\end{array}$ & $\begin{array}{l}90 \% \\
10 \%\end{array}$ \\
\hline 1959 & $\begin{array}{l}\text { O. fusiformis f. typica } \\
\text { o. fusiformis f. cornutogastra }\end{array}$ & $\begin{array}{r}98 \% \\
2 \%\end{array}$ & $\begin{array}{r}98 \% \\
2 \%\end{array}$ & $\begin{array}{r}98 \% \\
2 \%\end{array}$ \\
\hline 1960 & $\begin{array}{l}\text { o. fusiformis 1. typica } \\
\text { o. fusiformis f. cornutogastra }\end{array}$ & $\begin{array}{r}\mathbf{9 5 \%} \\
\mathbf{5} \%\end{array}$ & $\begin{array}{r}93 \% \\
7 \%\end{array}$ & $\begin{array}{r}93 \% \\
7 \%\end{array}$ \\
\hline $\begin{array}{c}\text { Santos } \\
1960\end{array}$ & $\begin{array}{l}\text { o. fusiformis 1. typica } \\
\text { o. fusiformis 1. cornutogastra }\end{array}$ & $\begin{array}{r}\mathbf{9 5} \% \\
\mathbf{5} \%\end{array}$ & $\begin{array}{r}93 \% \\
7 \%\end{array}$ & $\begin{array}{r}\mathbf{9 5 \%} \\
\mathbf{5} \%\end{array}$ \\
\hline
\end{tabular}

the total. The percentage of this species, decreases from station I to station III off Cananéia, but remains at aproximately the same level at the stations off Santos.

Two other species with relative abundance were $O$. fusiformis and $O$. dioica.
O. fusiformis was present in its two forms: typica and cornutogastra. Table $\mathrm{V}$ shows the abundance of cornutogastra in relation to typica. This species was more abundant at stations II and III than at station. I off Cananéia. Off Santos, it was more 
TABLE VI - Absolute numbers for each sample of the two forms of $F$. formica (f. tuberculata $\left({ }^{*}\right)$ and f. digitata (\$)

\begin{tabular}{|c|c|c|c|c|c|c|c|c|c|c|c|c|}
\hline Region & \multicolumn{9}{|c|}{ Cananéia } & \multirow{2}{*}{\multicolumn{3}{|c|}{$\begin{array}{c}\text { Santos } \\
1960\end{array}$}} \\
\hline Year & \multicolumn{3}{|c|}{1958} & \multicolumn{3}{|c|}{1959} & \multicolumn{3}{|c|}{1960} & & & \\
\hline Month Station & I & II & III & I & II & III & I & II & III & I & II & III \\
\hline Jan & - & - & - & - & $2^{*}$ & $2 *$ & $1 \S$ & - & $\begin{array}{r}53^{*} \\
246 \$\end{array}$ & - & $\overline{7}$ * & - \\
\hline Feb & - & - & - & - & - & $5^{*}$ & $8^{*}$ & $32^{*}$ & $791^{*}$ & - & $44 \S$ & $22 \S$ \\
\hline Mar & - & - & - & - & $7^{*}$ & $114 *$ & $64 *$ & $94^{*}$ & $106^{*}$ & 25 * & $19^{*}$ & $29^{*}$ \\
\hline Apr & - & - & - & - & - & - & - & $24^{*}$ & $45^{*}$ & $1^{*}$ & $18^{*}$ & $109^{*}$ \\
\hline May & - & - & - & - & - & - & - & - & - & - & - & - \\
\hline Jun & - & - & - & - & - & - & - & - & - & - & - & $92 \S$ \\
\hline Jul & - & - & - & - & - & - & - & - & - & - & $53^{*}$ & - \\
\hline Aug & - & - & - & - & - & - & - & - & - & $1 *$ & $11 *$ & $41 *$ \\
\hline Sep & - & - & - & - & - & - & - & - & - & - & $1 \&$ & $9 *$ \\
\hline Oct & - & - & - & - & - & - & - & - & $6 *$ & $39 *$ & $\begin{array}{r}203^{*} \\
23 \&\end{array}$ & $\begin{array}{l}38 * \\
27 \&\end{array}$ \\
\hline Nov & - & - & - & - & - & - & $2 *$ & - & $2^{*}$ & $6^{*}$ & $17 \S$ & $24^{*}$ \\
\hline Dec & - & - & $9^{*}$ & - & - & $28 *$ & - & - & $3^{*}$ & $16^{*}$ & $38^{*}$ & - \\
\hline
\end{tabular}

abundant at stations I and II. This fact seems to indicate that $O$. fusiformis prefers a certain distance from the coast.

O. dioica, showed a decrease in abundance from station I to station III of Cananéia. This species was present in greater number at station I off Santos. Therefore, it seems to be more neritic in habitat prefering to live close to the coast. Thus, station I off Santos and station III off Cananéia are different in relation to the occurrence of $O$. fusiformis and $O$. dioica (Almeida Prado, 1968).

The other species were most frequent at station III off Cananéia and at stations I, II and III off Santos. From these Fritillaria haplostoma was also present at stations I and II off Cananéia and during all the months sampled, f. typica considered oceanic form (ТокіокA, 1955) was most frequent than abjornseni considered a neritic form (BJöRNBERG \& FORNERIS, 1956a; TоKıоKA, 1956).

$O$. rufescens, $F$. borealis, $F$. formica, $F$. pellucida and $K$. tenuis were more abundant in January, February, March and April.

Two forms of $F$. borealis mainly f. intermedia and sargassi were found but always in different samples, the former more frequent in "shelf-water" and the latter in "tropical water" (ForNeris, 1965). Probably these two forms have different environmental requi- rements but no relationship could be established between these forms and the environmental parameters: temperature and salinity.

For $F$. formica the form tuberculata was more frequent than the form digitata. This latter form seems to be characteristic of the South Equatorial Current (Lohmann, 1896). Table VI shows the annual distribution of the two forms at stations off Cananéia and off Santos.

Kowalevskia tenuis occurred mostly in samples collected in "shelf waters".

Appendicularia sicula and Stegosoma magnum were rare.

c) Seasonal variation of $\mathrm{O}$. longicauda, $\mathrm{O}$. fusiformis and $\mathrm{O}$. dioica.

Since these three species constituted the bulk of the Appendicularia population it is worth giving their seasonal variation.

The seasonal variation of $O$. longicauda was irregular (Fig. 1). Since it is the dominant species it sets the pattern for the seasonal variation of the total Appendicularia population at the three stations off Cananéia and off Santos.

Seasonal occurrence of $O$. longicauda for the years 1958 - 1960 was similar at stations I and II off Cananéia. Thus at station I maximum numbers occurred in Summer - Fall and minimum in Spring. 


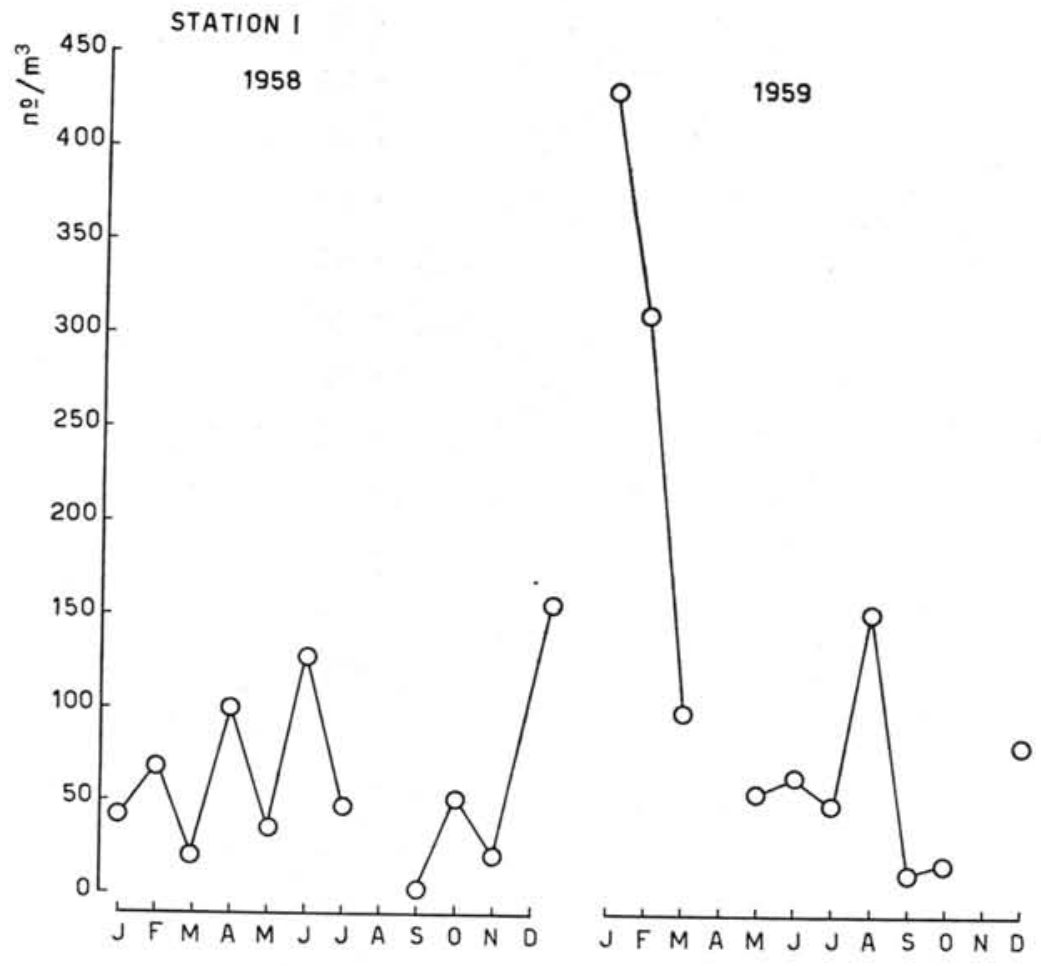

○
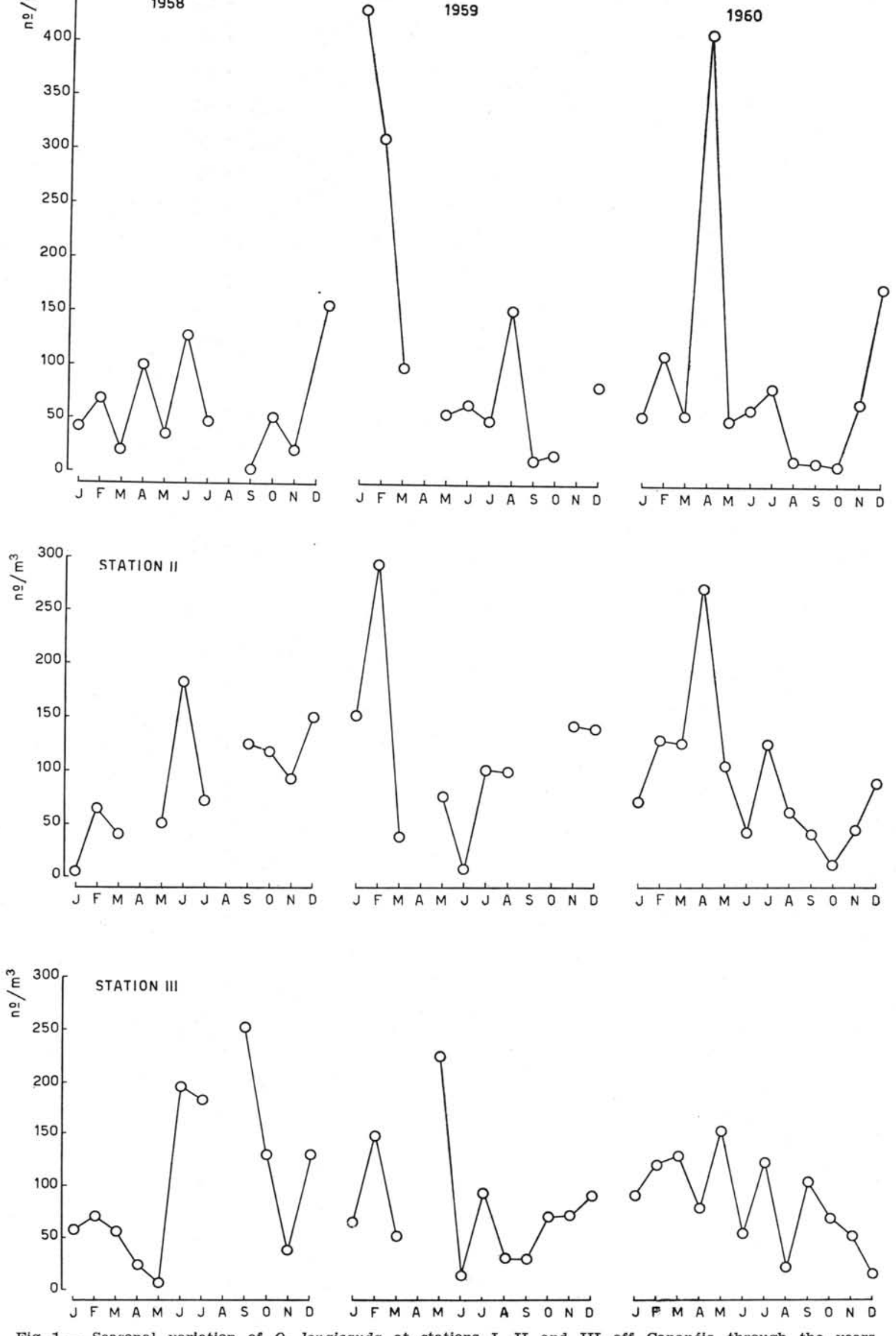

Fig. 1 - Seasonal variation of $O$. longicauda at stations I, II and III off Cananéla through the years 1958-1959-1960. 


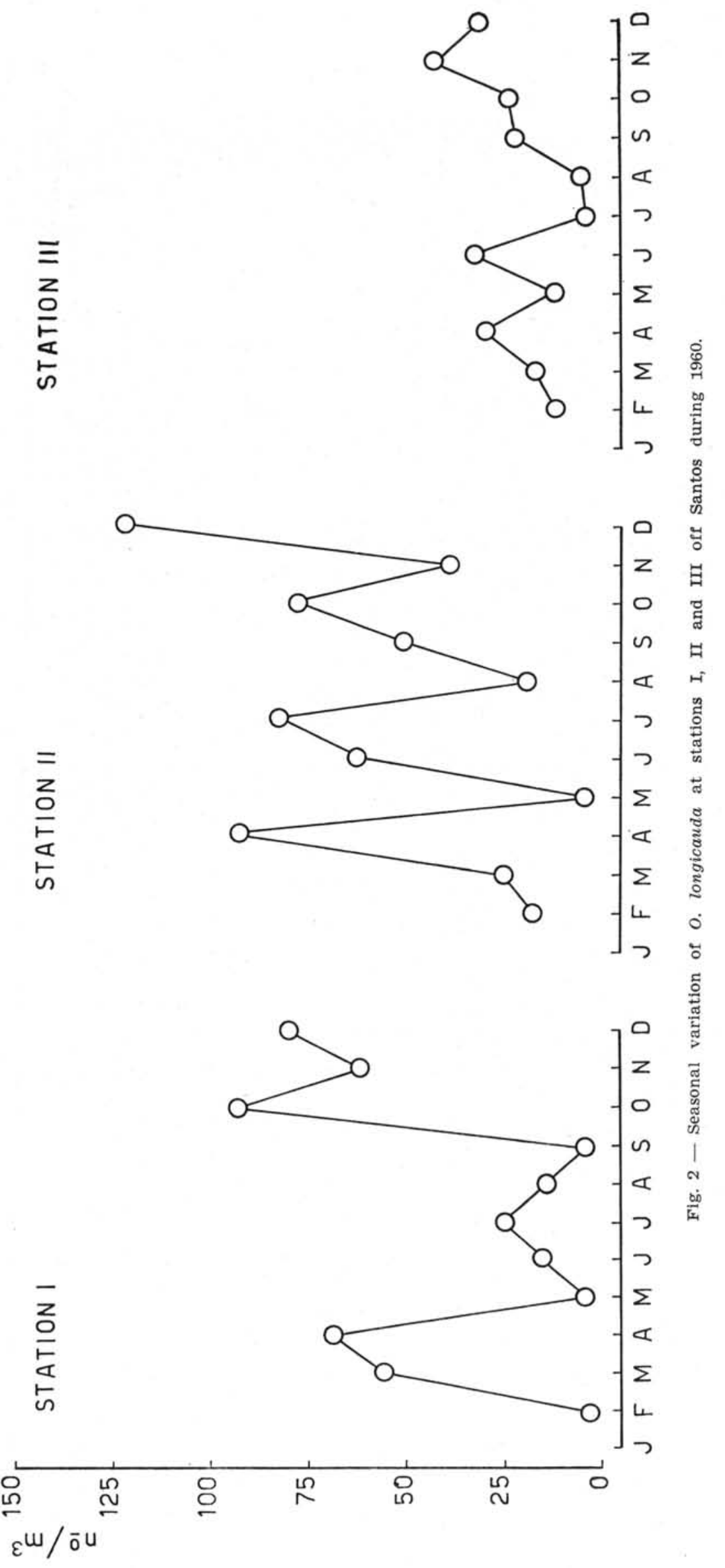




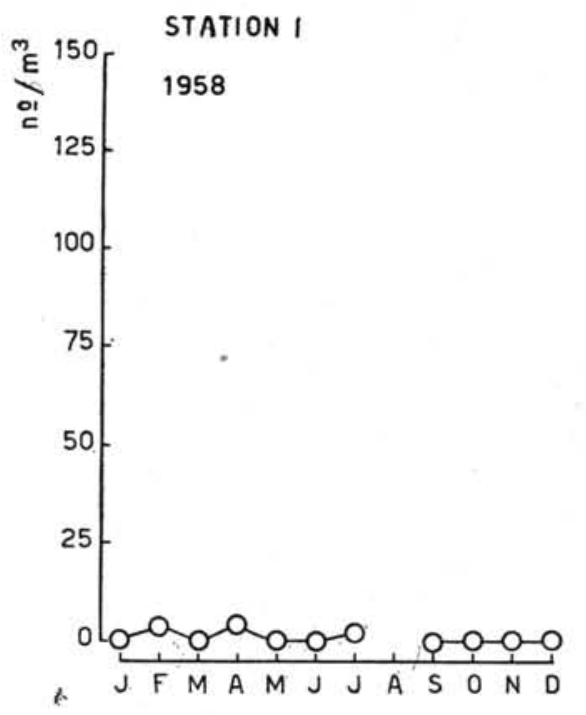

1959

1960
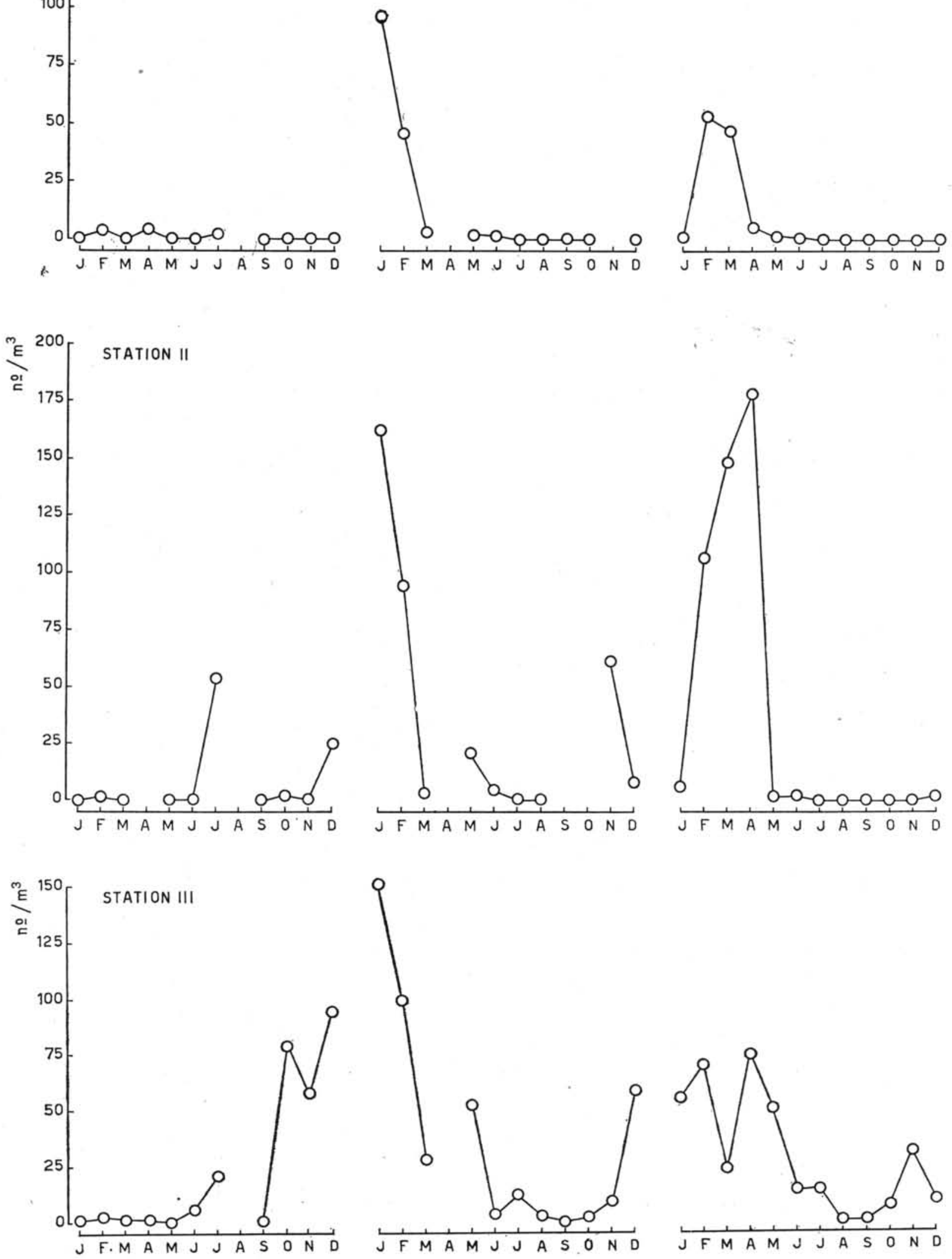

Fig. 3 - Seasonal variation of $O$. fusiformis at stations I, II and III off Cananéia through the years 1958-1959-1960. 


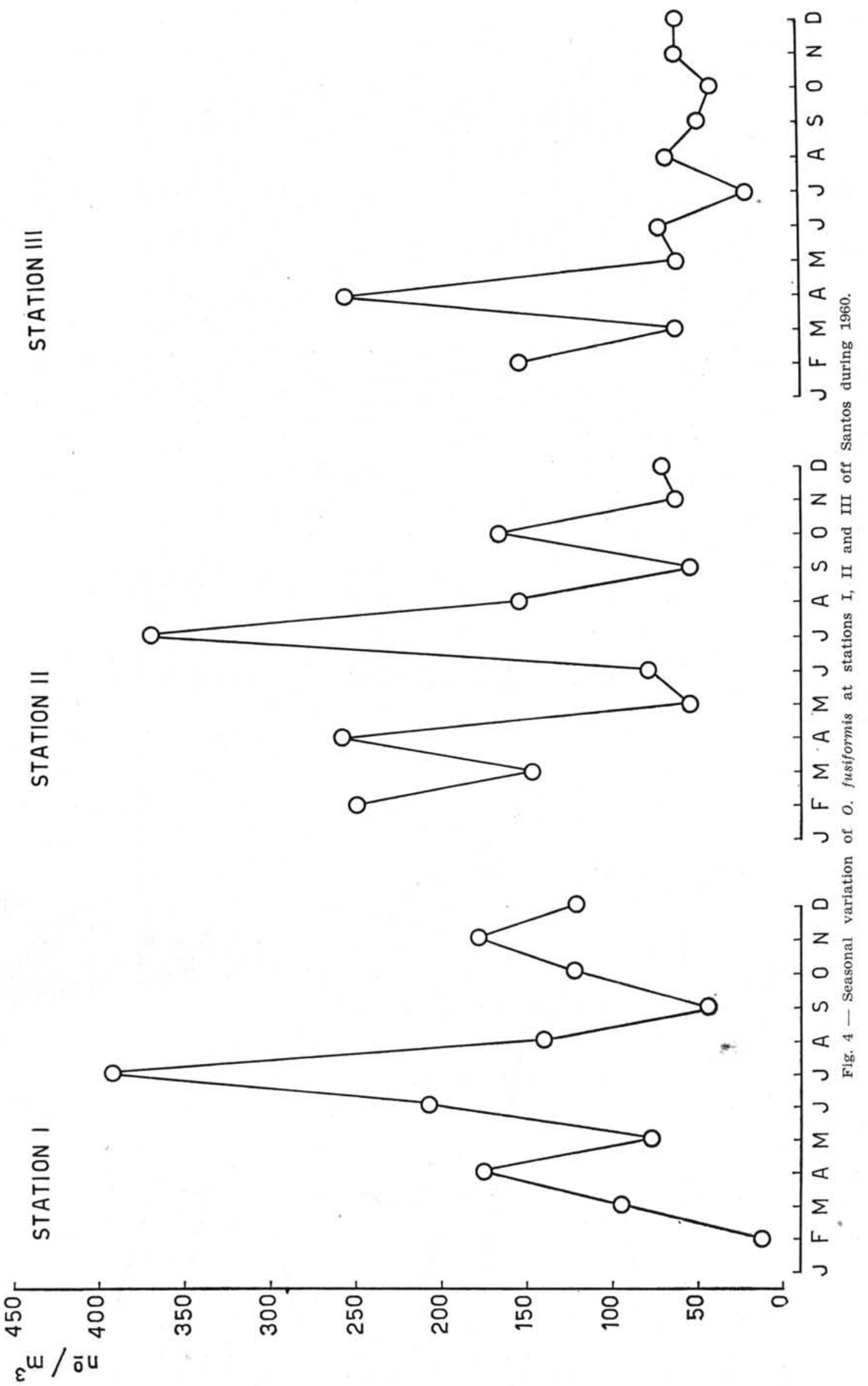



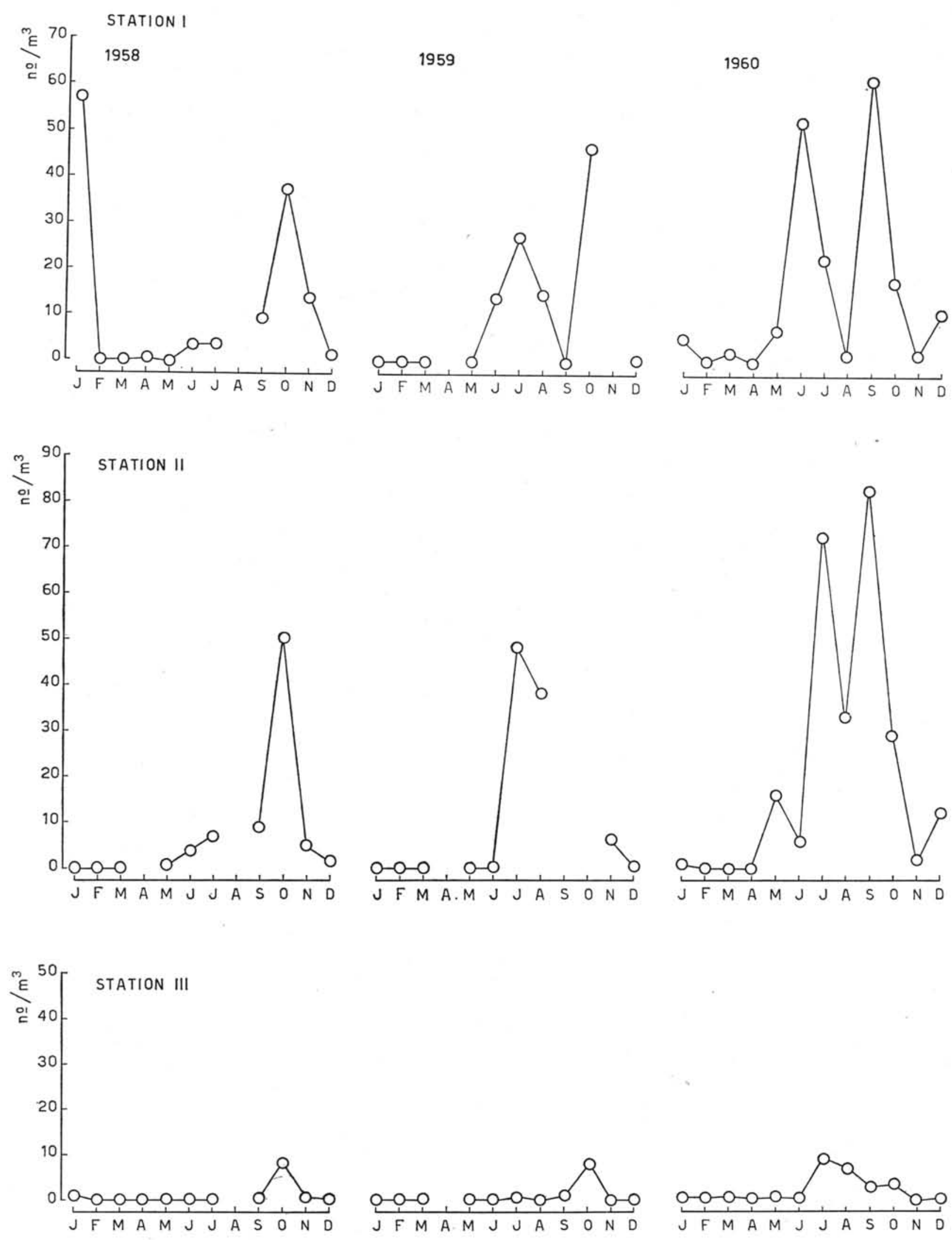

Fig. 5 - Seasonal variation of 0 . dioica

at stations I, II and III off Cananela through the years 1958-1959-1960. 


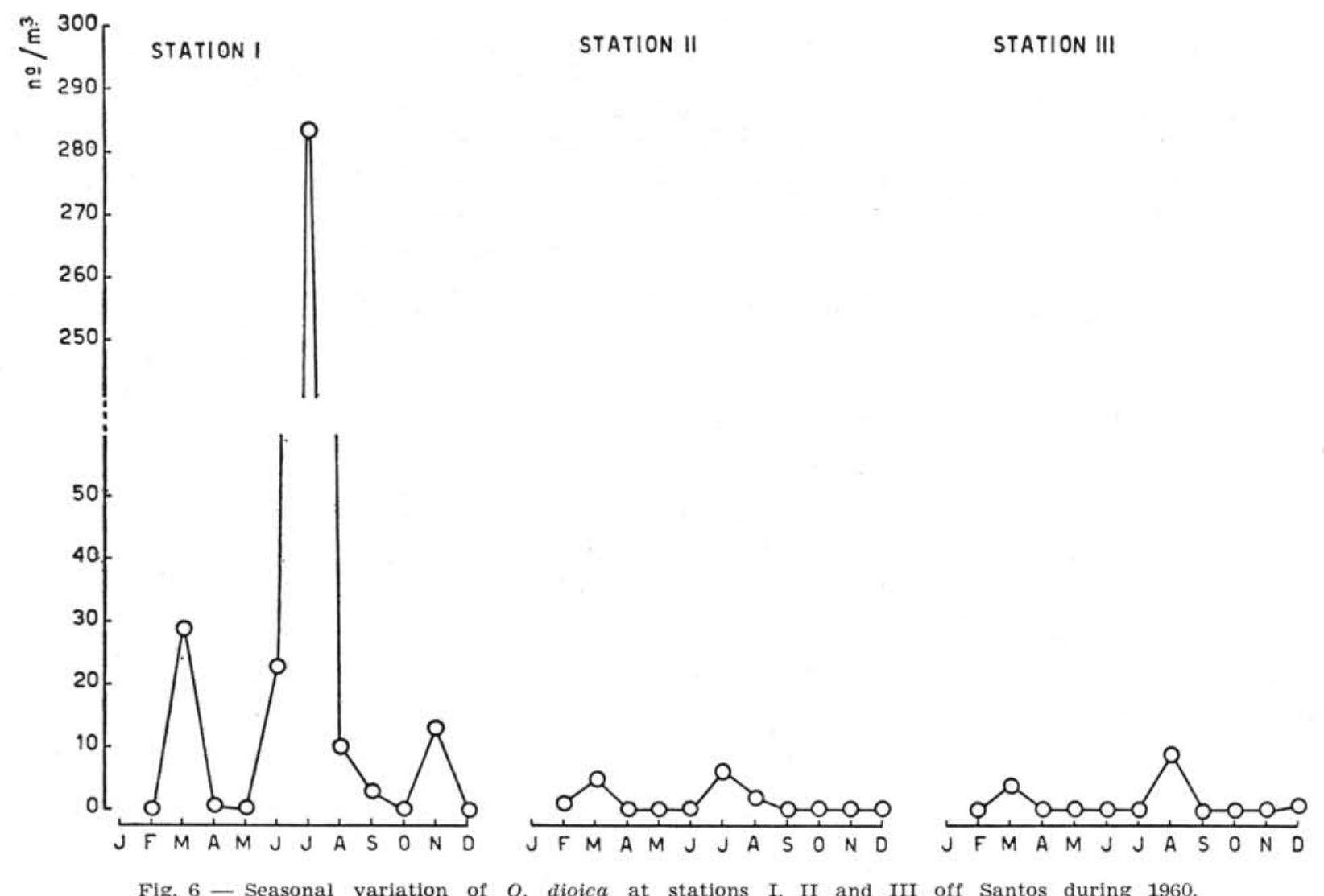

The same occurred at station II. At station III maximum numbers were found in September and May (1958 - 1959), minimum numbers in May (1958) and in June, August, September (1959). In 1960 the occurrence varies considerably all through the year.

The variation in number of individuals was greater at stations I and II than at station III, probably due to the fact that the latter station is a more stable environment.

Figure 2 shows the seasonal variation of $O$. longicauda at the three stations off Santos.

$O$. fusiformis and $O$. dioica showed a more regular pattern of seasonal variation.

The greatest density of $O$. fusiformis was found from November to February sometimes until April off Cananéia (Fig. 3). Off Santos its seasonal variation was slightly irregular particularly at station II (Fig. 4). This species has been characterized by many authors as a warm water dweller.

$O$. dioica is a neritic species, with a maximum number of individuals during July, September and October. A peak occurred eventually at station I in January (1958) off Cananéia (Fig. 5). Off Santos, maximum values occurred in June at station I (Fig. 6).

This species is characterized as common in coastal waters of relatively low salinity and estuaries (TокıокA, 1960; Tokioka \& Suarez CaAbro, 1956; Hopkins, 1966). It is the only species found at the lagunar region of Cananéia (Teixeira, Tundisi \& Kutner, 1965).

\section{d) Conclusions}

The region under study showed a small number of species comparing to other tropical or subtropical areas, probably due to the selectivity of environmental conditions. The three stations made both off Cananéia and off Santos were located very near to the coast and of small depth (maximum $49 \mathrm{~m}$ ) and are unfavourable for the capture of certain species, specially those of the genus Fritillaria, most usually encountered at greater depths or in regions far from the coast.

The abundance of Appendicularia recorded both for the stations in Santos and Cananéia is based almost entirely on the occurrence of $O$. longicauda, the dominant species in the region. The density values at the referent stations were similar. The little variation on the environmental conditions of the region under study probably would not affect the "stock" of the Appendicularia population.

The present study did not show a regular pattern of seasonal variation except for $O$. dioica and $O$. fusiformis, which however did not affect significantly the total number of individuals present. An irregular seasonal variation seems to be a common pattern for the plankton from the tropical and subtropical waters (HeINRICH, 1962). The present observations confirm this pattern for the abundance of Appendicularia. 


\section{ACKNOWLEDGEMENTS}

Thanks are due to Dr. Liliana Forneris for suggestions in the preparation of this paper. The author also, wishes to express her thanks to all the persons who have helped in its preparation.

\section{RESUMO}

Amostras coletadas quinzenalmente em três estações fixas ao largo de Cananéia durante os anos de 1958-1959-1960 e ao largo de Santos em 1960 foram analisadas para o estudo da variacão sazonal e composição qualitativa de Apendicularias.

$O$. longicauda foi a espécie dominante da região apresentando uma variação sazonal irregular a qual fo padrão para a variação do total da população de Apendicularias.

O. fusiformis e $O$. dioica apresentaram uma variacão sazonal mais regular, a primeira sendo mais abundante nos meses onde a temperatura da água foi mais elevada e a segunda nos meses onde a temperatura foi mais baixa.

\section{REFERENCES}

ALMEIDA PRADO, M. S. de

1968. Distribution and annual occurrence of Chaetognatha off Cananéia and Santos coast (São Paulo, Brazil) Bolm Inst. oceanogr. S Paulo, vol. 17, p. 35-55.

\section{BJÖRNBERG, T. K. S. \& FORNERIS, L.}

1956a. On the uneven distribution of the Copelata of the Fernando de Noronha area. Bolm Inst. oceanogr. S Paulo, vol. 7, n. $1 / 2$, p. 105-111.

1956b. On the uneven distribution of the Copelata of the Alcatrazes area. Bolm Inst. oceanogr. S Paulo, vol. 7, n.० 1/2, p. 113-115.

1958. Resultados científicos de los cruceros del "Baependi" y del "Vega" a la Isla Trindade. Copelata II. Neotropica, vol. 4, n. ${ }^{\circ} 15$, p. $81-85$.

EMILSSON, I.

1959. Alguns aspectos físicos e químicos das águas marinhas brasileiras. Ciênc. Cult. S Paulo, vol. 11 , n. ${ }^{\circ} 2$, p. 44-54.

1961. The shelf and coastal waters off Southern Brazil. Bolm Inst. oceanogr. S Paulo, vol. 11, n. 2 , p. 101-102.

FENAUX, R.

1959. Observations écologiques sur les appendiculaires du plancton de surface dans la baie de Villefranche-sur-Mer. Bull. Inst. océanogr. Monaco, n.o 1141, p. 1-26.

1963. Ecologie et biologie des appendiculaires méditerranéens (Villefranche-sur-Mer). Vie Milieu, suppl. n.॰ 16, p. 1-142.

1967. Les appendiculaires. Campagnes de la Calypso au large des côtes atlantiques de l'Amérique du Sud. Résult. scient. Camp. Calypso, fasc. 8, p. 44-46.
1969. Les appendiculaires du Golfe du Bengale. Expédition Internationale de l'Océan Indien (croisiéres du "Kistna", juin-aôut 1964). Mar. Biol., vol. 2 , n. ${ }^{\circ}$ 3, p. 252-263.

FORNERIS, L.

1965. Appendicularian species groups and Southern Brazil water masses. Bolm Inst. oceanogr. S Paulo, vol. 14, p. 53-114.

GARSTANG, W. \& GEORGESON, E.

1935. Report on the Tunicata. Part II. Copelata. Br. Antarct. "Terra Nova" Exped. 1910, Zool. 4, p. 263-282.

HEINRICH, A. K.

1962. The life histories of plankton animals and seasonal cycles of plankton communities in the oceans. J. Cons, perm. int. Explor. Mer., vol. 27, p. 15-24

HOPKINS, L. T.

1966. The plankton of the St. Andrew Bay system, Florida. Publs Inst, mar. Sci. Univ, Tex., vol. 11, p. 1-64.

LEINEBÖ, R.

1969. Study of coastal water on the Brazilian shelf at latitude $25^{\circ} \mathrm{S}$. Contrçôes Inst. oceanogr. Univ. S Paulo, sér. Ocean. fís., n. ${ }^{\circ} 11$, p. $1-14$

LOHMANN, $\mathrm{H}$.

1896 Die Appendicularien der Plankton Expedidition. Ergebn. Atlant. Ozean Planktonexped. Humboldt-Stift., vol. 2 E C., p. 1-148.

1931. Die Appendicularien der Deutschen Tiefsee-Expedition. Wiss. Ergebn.dt.TiefseeExped. "Valdivia", vol. 21, n. 1, p. 1-158.

LOHMANN, H. \& BÜCKMANN, A.

1926. Die Appendicularien der Deutschen Südpolar Expedition 1901-03. Dt. Südpol.Exped., vol. 18, (Zool. 10), p. 63-231.

LOHMANN, H. \& HENTSCHEL, E.

1939. Die Appendicularien im Südatlantischen Ozean. I. Die Horizontalverbreitung der Wichtigsten Arte. Wiss. Ergebn. dt. atlant. Exped. "Meteor", vol. 3, n..$^{\circ}$, p. 154-182.

TAVARES, D. Q.

1967. Occurrence of doliolids and salps during 1958, 1959 and 1960 off the São Paulo coast. Bolm Inst oceanogr. § Paulo, vol. 16 , p. $87-97$.

TEIXEIRA, C., TUNDISI, J. \& KUTNER, M. B.

1965. Plankton studies in a mangrove environment. II. The standing-stock and some ecological factors. Bolm Inst. oceanogr. S Paulo, vol. 14, n. ${ }^{\circ}$ 1, p. 13-41.

TOKIOKA, T.

1955. General consideration on Japanese appenGeneral consideration on Japanese appen-
dicularian fauna. Publs Seto mar. biol. Lab., vol. 4 , n. 2/3, p. 251-261. 
1956. On chaetognaths and appendicularians collected in the central part of the Indian Ocean. Publs Seto mar. biol. Lab., vol. 5, n. ${ }^{\circ} 2$, p. 197-202.

1960. Studies on the distribution of appendicularians and some thaliaceans of the North Pacific with some morphological notes. Publs Seto mar. biol. Lab., vol. 8 , n. 2 , p. $351-443$.
TOKIOKA, 'T. \& SUÁREZ CAABRO, J. A.

1956.

Apendicularias de los mares cubanos. Mems Soc. cub. Hist. nat. "Felipe Poey", vol. 23, n. 1 , p. $37-80,15$ pls.

UDVARDY, M. D. F.

1958. Appendicularia. Further zool. Results Swed. Antarct. Exped., vol. 5, n. ${ }^{\circ}$ 1, p. 2-15. 